\title{
Overlap and Differences in Brain Networks Underlying the Processing of Complex Sentence Structures in Second Language Users Compared with Native Speakers
}

\author{
Kirsten Weber, ${ }^{1,2}$ Lisa Luther, ${ }^{2}$ Peter Indefrey, ${ }^{2,3}$ and Peter Hagoort ${ }^{1,2}$
}

\begin{abstract}
When we learn a second language later in life, do we integrate it with the established neural networks in place for the first language or is at least a partially new network recruited? While there is evidence that simple grammatical structures in a second language share a system with the native language, the story becomes more multifaceted for complex sentence structures. In this study, we investigated the underlying brain networks in native speakers compared with proficient second language users while processing complex sentences. As hypothesized, complex structures were processed by the same large-scale inferior frontal and middle temporal language networks of the brain in the second language, as seen in native speakers. These effects were seen both in activations and task-related connectivity patterns. Furthermore, the second language users showed increased task-related connectivity from inferior frontal to inferior parietal regions of the brain, regions related to attention and cognitive control, suggesting less automatic processing for these structures in a second language.
\end{abstract}

Key words: activation; bilingualism; connectivity; fMRI; language; PPI; syntax

\section{Introduction}

W HEN WE LEARN A SECOND LANGUAGE later in life, we already have an entire first language system in place. There is an established system, a brain network, for processing sounds, words, and sentences in this language. As we start learning a second language, it is intuitively plausible that we recruit parts of the same brain network that is already established for the first language. Indeed most meta-analysis studies on second language processing in the brain confirm this idea (Indefrey, 2006) at least if participants are proficient in their second language (Sebastian et al., 2011). These studies largely focused on simple sentence structures that overlap in structure with those in the first language. However, we know less about the networks engaged in processing complex structures and especially those structures that have no direct correspondents in the first language.

A structure that does not exist in the same form in the first language cannot be tested across languages in the same subjects. In this study, we therefore compared the processing of complex sentence structures in a group of second language users to a group of native speakers of Dutch. We investigated two types of complex sentence structures, one that existed in the first language of the second language user group (a rightbranching structure) and one that did not (a crossed dependency structure).

Crossed dependencies (Fig. 1A) are complex sentence structures that are infrequent in their appearance across the languages of the world, Dutch and Swiss-German being among the few. In standard German, a nested dependency structure would be used instead (Fig. 1C). These sentences look very similar: both have nonlocal dependencies and only the final verbs appear to be swapped. Nonetheless, linguistically, they belong to different types of syntactic classes (Nowak et al., 2002). The crossed dependency structure is generated by context-sensitive grammars, while the nested dependency structure is generated by the lower class of context-free grammars. Psycholinguists have discovered differences in the processing of these structures (Bach et al., 1986; Kaan and Vasić, 2004). However, contrary to the linguistic hierarchy, the crossed dependencies appear to be easier to process than the nested dependency structures. Therefore, both from a linguistic and a psycholinguistic perspective, crossed dependency structures are different from nested dependency structures in their syntactic structure, while the meaning conveyed is similar.

\footnotetext{
${ }^{1}$ Max Planck Institute for Psycholinguistics, Nijmegen, The Netherlands.

${ }^{2}$ Donders Centre for Cognitive Neuroimaging, Donders Institute for Brain, Cognition and Behaviour, Radboud University Nijmegen, Nijmegen, The Netherlands.

${ }^{3}$ Department of Linguistics, Heinrich-Heine University, Duesseldorf, Germany.
} 


\section{A Dutch: Crossed-Dependency}

FIG. 1. Examples of complex sentence structures in German and Dutch and the dependencies between verbs and arguments. The structures in $(\mathbf{A}, \mathbf{B})$ are used in the present study. The structure in (A) does not exist in standard German and an embedded structure, see (C), is possible instead; $(\mathbf{B}, \mathbf{D})$ on the other hand are equivalent in German and Dutch. All sentences mean "Jan taught Anna to feed the horses."

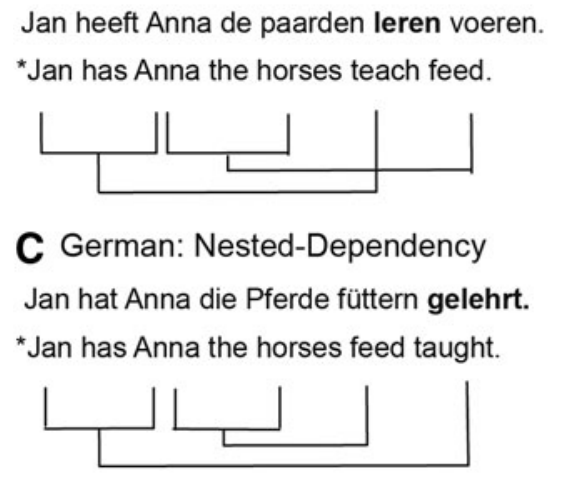

Jan heeft Anna de paarden leren voeren. Jan has Anna the horses teach feed.

C German: Nested-Dependency

Jan hat Anna die Pferde füttern gelehrt.

an has Anna the horses feed taught.
B Dutch: Right-Branching

Jan heeft Anna geleerd de paarden te voeren. *Jan has Anna taught the horses to feed.

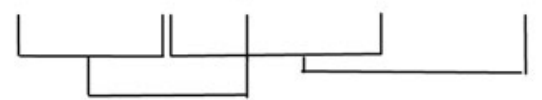

D German: Right-Branching

Jan hat Anna gelehrt die Pferde zu füttern.

*Jan has Anna taught the horses to feed.

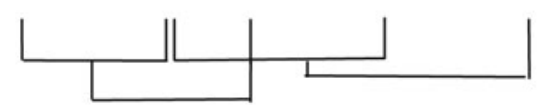

The processing of crossed dependencies in second language speakers is thus an interesting test bed to investigate how these later learned complex structures are processed in the brain. Right-branching sentence structures (Fig. 1B, D) on the other hand are structures with a similar meaning to the sentences in Figure $1 \mathrm{~A}$ and $\mathrm{C}$, but with an equivalent structure across the two languages. Moreover, while still complex structures, they are thought to be easier to process than embedded structures (Stromswold et al., 1996).

While crossed dependencies do not exist in German, German native speakers can nonetheless acquire and understand these structures rapidly, even in adulthood (Davidson and Indefrey, 2009; Uddén et al., 2012), as evidenced by behavioral and electrophysiological measures. Accordingly, one might assume that the same hemodynamic effects should be found whether the structure is processed by a first language speaker or second language user.

In the initial analysis of the present dataset published in Weber's PhD thesis (2012), syntactic hemodynamic repetition effects of crossed dependency structures in a first language speaker group were compared with the repetition effects of the same structures in a second language (L2) user group. This analysis showed subtle differences in the repetition effects between the two groups. However, these effects, while different across groups (the native speaker group showed a repetition enhancement effect and not the expected repetition suppression effect, while the German group did not), were not in the expected direction and moreover very subtle, only present in a region of interest analysis (focusing on the left inferior frontal gyrus and left and right temporal cortex).

While this hints at a slightly different organization of the processing systems within the same brain regions, in the present analysis, we were interested in figuring out whether the overall brain activation patterns and networks differed across the two groups. More specifically, we were interested in discovering whether the overall language network was larger in the L2 user group or whether the networks completely overlap at the whole-brain level. In this article, we thus report a complementary classic activation and functional connectivity analysis of this dataset to elucidate whether differences in hemodynamic responses between the two groups are confined to the core regions of the language network or whether the language network is organized differently for processing the same language as an L1 or as an L2.

According to some theories, later learned syntactic structures should at least initially be processed by a different brain network. Ullman (2001a) proposed that L2 speakers rely more on a declarative system to process the second language, while L1 processing is more procedural. Two different neural networks, that is, a left frontotemporal and a frontostriatal network are proposed to underlie these processes, respectively (Ullman, 2001b). Both are claimed to involve Broca's area (Ullman, 2006).

Others propose that while the overall network for grammatical processing is very similar for the first and second language, there are activation differences. Namely, L2 speakers engage more regions if the L2 was acquired later in life and specifically if they are less proficient in the L2. This pertains especially to regions related to cognitive control, such as those regions related to suppressing interfering lexical or syntactic representations from the other language (Abutalebi et al., 2008; Green and Abutalebi, 2013). Other accounts suggest differences in the processing of local and nonlocal dependencies (Clahsen and Felser, 2006; Dallas and Kaan, 2008). In case of local dependencies, L2 grammatical processing can become native-like. However, the processing of complex syntax, such as nonlocal dependencies, might differ (Clahsen and Felser, 2006).

Yet, others claim that the same brain network processes both the first and the second language (Consonni et al., 2013; Indefrey, 2006; Luke et al., 2002; Perani et al., 1998). As indicated previously, stronger evidence for a shared syntactic system between L1 and L2 comes from a repetition suppression study in German-English late bilinguals (Weber and Indefrey, 2009). The study showed that an English passive can be primed by a German passive on both the neural and the behavioral levels. Therefore, it was argued that the same neuronal populations were recruited in the processing of the L1 and the L2 passive. Similar behavioral effects in language production have been found in Spanish-English and Dutch-English bilinguals (Hartsuiker et al., 2004; Schoonbaert et al., 2007). Most of these studies focused on sentences with simple local dependencies. It is thus an open question whether advanced second language users process these later learned complex structures like native speakers or whether they rely on different processes and representations.

In the past, functional neuroimaging studies of language processing have focused on activation studies alone, trying to investigate where a certain cognitive function is localized. However, this approach does not take the dynamic interplay between regions during cognitive tasks into account 
(Hagoort, 2014). Therefore, investigations of functional, task-related connectivity patterns are an important addition to elucidate the large-scale networks underlying language processing. Recent studies have shown the dynamic interplay between inferior frontal and temporal regions during language processing (den Ouden et al., 2012; Griffiths et al., 2013; Snijders et al., 2010).

The left inferior frontal and temporal regions of the brain are thought to form the core network related to sentence and especially syntactic processing as described by several different theories (e.g., Grodzinsky and Friederici, 2006; Hagoort, 2005) and as evidenced, for example, by syntactic priming studies (Menenti et al., 2011; Segaert et al., 2012). This functional organization appears to be realized across different languages, in closely related languages such as German and Dutch, as well as in less related languages such as Japanese, Chinese, and Hebrew (see e.g., the studies listed in the supplementary materials of a recent meta-analysis of sentence-level processing, Hagoort and Indefrey, 2014).

In the present study, we are going to investigate the language networks of native speakers and second language users and investigate, with activation and connectivity analyses of functional magnetic resonance imaging (fMRI) data, whether the networks overlap between the two groups. Moreover, we are going to explore whether there are any overall group differences in activation and connectivity patterns and, more specifically, whether there are any network differences between the two groups for the processing of crossed dependency structures, the structure that does not exist in the first language of the second language user group (in comparison with the right-branching structure that does occur in both languages).

We expect these differences to show up in the form of and increased engagement of the brain networks in L2 processing, in the sense of more activation and more connectivity (either in the form of additional network nodes or stronger connections) within the underlying networks. These differences might occur within the traditional language networks thought to underlie sentence and, in particular, syntactic processing (i.e., left inferior frontal and left middle temporal cortex), which would indicate differences in the nature of the second language networks, or in other nontraditional regions that are, for example, linked to cognitive control mechanisms on the language network (such as prefrontal or parietal regions of the brain).

\section{Materials and Methods}

\section{Participants}

We tested 24 Dutch native speakers ( 15 females) and 28 German native speakers (18 females). Four of the German participants (three females) were subsequently excluded as they did not meet our criteria concerning their language background or due to technical malfunction during scanning. One Dutch native speaker was excluded from the analyses as the condition triggers were not recorded correctly. The German native speakers all went to university in The Netherlands and had started learning Dutch after the age of 18 . They had all passed the Dutch NT2 staatsexamen, a language test that allows university entry and shows a high-proficiency level in Dutch. All participants were right-handed and had no history of neurological impairments. They all had normal or corrected-to-normal vision. The participants received course credits or money for their participation in the experiment. All participants gave written informed consent before the study started and the study was conducted according to institutional guidelines of the local ethics committee (CMO protocol region Arnhem-Nijmegen, The Netherlands) and in accordance with the Declaration of Helsinki.

\section{Stimuli and experimental design}

The experimental stimuli consisted of crossed dependency sentences and right-branching structures with similar semantic content (Fig. 1A, B). The study was originally designed as a syntactic priming experiment in which crossed dependency structures were primed (syntactic priming trial: prime: crossed dependency structure, target: crossed dependency structure; no syntactic priming trial: prime: right-branching structure, target: crossed dependency structure). This was fully crossed with a verb repetition manipulation. The main verbs used in the sentences were helpen (to help) and leren (to teach) (crossed dependency structures in Dutch are restricted to a very limited set of main verbs [Zwart, 1996] and we thus used only two).

Instead of comparing target sentences as in the original analysis, here we look at the orthogonal contrasts of crossed dependency and right-branching prime sentences. Given the design, we thus have equal numbers (52) of crossed dependency and right-branching sentences and an even distribution of the two verbs. The original description of the design can be found in Weber (2012). Moreover, to hide the priming manipulation and make the sentences less predictable, an equivalent amount of passive and active filler trials, as well as right-branching filler trials, was also presented to the participant interspersed with the priming trials. As a baseline condition, miniblocks of, in total, 64 sentence format consonant strings after every 20 sentences were presented as well. Four stimulus lists were created. Across these stimulus lists, each target occurred in a prime and a no-prime trial and each sentence's content appeared in the prime as well as target position. Each participant was presented with only one of the stimulus lists. The experiment consisted of three experimental sessions; participants had a short break between sessions.

Right-branching and crossed dependency sentences are not equal in numbers of words as the right-branching sentence has an additional word, te (Fig. 1). Moreover, an additional manipulation of the sentences that was originally conceived to ensure that syntactic priming effects cannot be explained by overlap in length between crossed dependency sentences, but no overlap with right-branching sentences, introduced an additional adjective in the second noun phrase of right-branching sentences (thus making these always 11 words long) as well as in the crossed dependency target sentences (thus 10 words long). No additional adjectives were introduced in crossed dependency prime sentences; these were thus nine words long.

Thus, our present comparison between right-branching and crossed dependency structures is confounded by length (9 versus 11 words) and additional adjectives in one condition. However, given our hypothesis, we are only interested in the interaction between participant group and type of structure as well as the main difference between groups, contrasts that should only be affected by this confound between the type of structures if the sentence length per se was processed differently in the two groups, which is unlikely. 


\section{fMRI experimental procedure}

The experiments were run using Presentation software (Neurobehavioral Systems, www.neuro-bs.com). Participants lay in the scanner and looked at a screen through a mirror. Sentences were presented in white Arial font of size 22 on a black background and participants were instructed to read the sentences silently in their head. Crossed dependency sentences were presented in these fragments: "De muzikant/ heeft/de tiener/de fluit/leren/spelen" (The musician has the teenager the flute teach play.) and right-branching sentences in these: "De muzikant/heeft/de tiener/geleerd/de fluit/te spelen" (The musician has the teenager taught the flute to play.). Sentence fragments were presented one by one at a fixed presentation rate that depended on the length of the fragment, both right-branching and crossed dependency sentences were presented in six fragments (while the number of words differed, see previous section).

The fragment duration in msec was computed as ([number of letters in the fragment $\times 30 \mathrm{msec}]+190 \mathrm{msec}$ ), a method based on Nieuwland and Van Berkum (2006). During the interstimulus interval (between sentences), a fixation cross was displayed. The length of the interval was jittered between 1.1 and $3.3 \mathrm{sec}$. In $13.3 \%$ of the nontarget experimental sentences and one third of the filler sentences, a word appeared in a larger 30 font size; these trials were not included in the current analysis. Subjects had to respond to this change by pressing a button.

\section{fMRI data acquisition and analysis}

The fMRI data were acquired on a 1.5 Tesla Siemens Avanto scanner. A functional T2*-weighted EPI-BOLD fMRI scan was performed $(\mathrm{TR}=2.35 \mathrm{sec}, \mathrm{TE}=40 \mathrm{msec})$, with a flip angle of $90^{\circ}$. We acquired 33 slices with a voxel size of $3.5 \times 3.5 \times 3 \mathrm{~mm}$ with a 0.5 -mm gap. The field of view was $224 \times 224 \times 115.5 \mathrm{~mm}$. The slices were acquired in an ascending order. It was made sure that the field of view included inferior parts of frontal and temporal cortex. In some subjects, parts of the top of the brain were outside the field of view. The anatomical images were acquired using a T1-weighted sequence. The fMRI data were preprocessed and analyzed at the first level using SPM5 (www.fil.ion.ucl.ac.uk/spm/).

Second-level analyses were run using SPM8. The first five images were discarded to ensure that transient nonsaturation effects did not affect the analysis. The functional images were checked for spikes and if any were detected these images were removed and a replacement image was created based on the surrounding images. All functional images were realigned and slice-time corrected. The participants' anatomical T1 images were coregistered to the mean functional image. The anatomical T1 images were then segmented into gray and white matter and the spatial normalization parameters were taken to normalize the functional images. Functional images were smoothed with a $10-\mathrm{mm}$ full-width at half maximum Gaussian kernel.

For the fMRI activation analysis, we first defined the contrasts of interest for each subject. These were then taken to the second level for a random-effects group analysis. Connectivity analyses were run using the generalized psychophysiological interaction (gPPI) toolbox (McLaren et al., 2012). As for the activation analysis, as per psychophysio- logical interaction analysis (thus per seed), we first defined the contrasts of interest for each subject. These were then taken to the second level for a random-effects group analysis.

\section{First-level single-subject model activation}

For the activation analyses, we created a design matrix for each participant that included for each of the three sessions, regressors for each type of sentence structure and the sentence format consonant strings, as well as the fixation cross. The actual event duration was modeled and realignment parameters for movement correction were added to the model. In addition, additional regressors were entered to covary for excessive movement at time points where composite motion was $>1 \mathrm{~mm}$. These covariates were created using the ART toolbox (Mozes and Whitfield-Gabrieli, 2011; www.nitrc.org/ projects/artifact_detect/). On average, the additional regressors were added for $<3 \%$ of the time points. We then defined three different contrasts per subject, the crossed dependency sentences, the right-branching sentences, and both these complex sentence structures, all against the baseline of sentence format consonant strings. These contrasts were taken to the second level for a random-effects group analysis.

\section{First-level single-subject model connectivity}

We also carried out task-related connectivity analyses from seeds that were defined based on second-level activation analyses. More specifically, based on the first six peaks of activation from the conjunction of complex sentences across the two groups (Table 1), we defined 10-mm spheres that were used as seeds for a gPPI analysis (McLaren et al., 2012). These seeds were located in the left inferior frontal gyrus, left temporal pole, left anterior middle temporal gyrus, left middle temporal gyrus, left posterior middle temporal gyrus, and left inferior temporal gyrus.

For each seed region and subject, we built a design matrix consisting of the following regressors: (1) regressors for each condition, mirroring the activation model; (2) a regressor for the overall activity in the seed region; and (3) regressors describing the modulation of activity from the seed region to the rest of the brain for each of the conditions (psychophysiological interactions). Moreover, the same regressors for movement correction as for the activation models were used. The actual event duration was modeled. We again defined three different contrasts of interests to be taken into the second-level group analysis: the modulation of activity from the seed region for crossed dependency sentences, rightbranching sentences, and both complex sentences against sentence format consonant strings.

\section{Second-level model activation}

To investigate overlap and differences in activation patterns between the two groups, we built two different secondlevel models. First, we built a flexible factorial model with the factors group (second language user; native speaker), type of sentences (crossed dependency or right branching), and subject in which we modeled both main effects and the interaction. Second, a simple between-groups $t$-test compared complex sentences versus sentence format consonant strings across the two groups. This was built as an additional model to be able to look at the main effect of the group, which is not correctly estimated in the flexible factorial 
Table 1. Activation and Connectivity Effects of the Conjunction of Sentences Versus Consonant Strings Contrast Between the Two Groups

\begin{tabular}{|c|c|c|c|c|c|c|}
\hline Region & $\begin{array}{l}\text { Cluster } \\
\text { size }\end{array}$ & $x / y / z$ & $\begin{array}{c}B A \\
\text { area }\end{array}$ & $\begin{array}{c}p_{F W E} \\
\text { (cluster) }\end{array}$ & $\begin{array}{l}p_{F W E} \\
(\text { peak })\end{array}$ & $T_{45}$ \\
\hline \multicolumn{7}{|l|}{ Activations } \\
\hline Left middle temporal gyrus ${ }^{\mathrm{a}}$ & 7725 & $-66-408$ & $21 / 22$ & 0.000 & 0.000 & 27. \\
\hline Left middle temporal gyrus (anterior) ${ }^{\mathrm{a}}$ & & $-62-6-10$ & 21 & & 0.000 & 25.4 \\
\hline Left medial temporal pole ${ }^{\mathrm{a}}$ & & $-4412-20$ & 38 & & 0.000 & 16.3 \\
\hline Left inferior temporal gyrus ${ }^{\mathrm{a}}$ & & $-38-12-28$ & 20 & & 0.000 & 15.1 \\
\hline Left middle temporal gyrus (posterior) ${ }^{\mathrm{a}}$ & & $-44-5218$ & 21 & & 0.000 & 13.1 \\
\hline Left inferior frontal gyrus orbitalis/triangularis ${ }^{\mathrm{a}}$ & & $-4628-4$ & $45 / 47$ & & 0.000 & 10. \\
\hline Left hippocampus & & $-24-24-12$ & & & 0.000 & \\
\hline Left fusiform gyrus & & $-38-38-18$ & 37 & & 0.000 & 7. \\
\hline Left rolandic operculum & & -62014 & 6 & & 0.000 & \\
\hline Left inferior frontal gyrus triangularis & & -582416 & 45 & & 0.000 & 6.8 \\
\hline Left superior temporal gyrus & & $-48-3224$ & 41 & & 0.01 & \\
\hline Left insula & & $-34-1814$ & & & 0.114 & \\
\hline Right middle temporal gyrus & 573 & $620-14$ & 21 & 0.000 & 0.000 & \\
\hline Right middle temporal pole & & $4818-26$ & 38 & & 0.000 & 7. \\
\hline Right middle/superior temporal gyrus & & $52-14-2$ & 22 & & 0.462 & \\
\hline Right (para)hippocampal gyrus & 417 & $22-14-16$ & 35 & 0.000 & 0.000 & 9.8 \\
\hline Left cerebellum & 373 & $-6-500$ & & 0.000 & 0.000 & \\
\hline Left precentral gyrus & 151 & $-52-646$ & 6 & 0.000 & 0.000 & 8.6 \\
\hline Left calcarine gyrus & 235 & $-2-9414$ & 18 & 0.000 & 0.001 & \\
\hline Cuneus & & $0-8636$ & 19 & & 0.073 & 5.0 \\
\hline Right cerebellum & 25 & $18-82-38$ & & 0.004 & 0.003 & \\
\hline Right precentral gyrus & 17 & $60-846$ & 6 & 0.008 & 0.007 & 5.9 \\
\hline \multicolumn{7}{|l|}{ (1) Connectivity from left inferior frontal gyrus } \\
\hline Left anterior superior temporal gyrus/left temporal pole & 78 & $-542-8$ & 38 & 0.029 & 0.005 & \\
\hline Left inferior occipital gyrus & 115 & $-26-88-8$ & 18 & 0.009 & 0.015 & \\
\hline Right inferior frontal cortex/insula & 77 & 322814 & & 0.030 & 0.076 & \\
\hline Left fusiform gyrus & 79 & $-42-60-10$ & 37 & 0.028 & 0.163 & \\
\hline \multicolumn{7}{|l|}{ (2) Connectivity from left temporal pole } \\
\hline Cerebellar vermis (lobule VI) & 77 & $2-78-20$ & & 0.028 & 0.198 & \\
\hline Left lingual gyrus & & $-16-84-10$ & 18 & & 0.601 & 4.23 \\
\hline \multicolumn{7}{|l|}{ (3) Connectivity from left anterior temporal gyrus } \\
\hline Right inferior frontal gyrus (opercularis) & 402 & 36630 & 44 & $0.000^{\mathrm{b}}$ & 0.000 & \\
\hline Left middle temporal gyrus & 464 & $-60-466$ & 21 & $0.000^{\mathrm{b}}$ & 0.000 & \\
\hline Left fusiform gyrus & 849 & $-42-52-16$ & 37 & $0.000^{\mathrm{b}}$ & 0.001 & \\
\hline Left middle occipital gyrus & & $-38-864$ & 19 & & 0.005 & \\
\hline Left lingual gyrus & & $-18-86-10$ & 18 & & 0.040 & \\
\hline Left superior occipital gyrus & 177 & $-26-7628$ & 19 & $0.002^{\mathrm{b}}$ & 0.002 & \\
\hline al gyrus & 829 & $28-7230$ & 19 & $0.000^{\mathrm{b}}$ & 0.003 & \\
\hline Right inferior occipital gyrus & & $44-860$ & 19 & & 0.007 & \\
\hline Right middle occipital gyrus & & $34-9020$ & 19 & & 0.175 & \\
\hline Right inferior occipital gyrus & & $40-62-10$ & 37 & & 0.323 & \\
\hline Left fusiform gyrus & & $40-62-10$ & 37 & & 0.323 & \\
\hline Left anterior/middle cingulate cortex & 119 & -10428 & 24 & $0.008^{\mathrm{b}}$ & 0.039 & \\
\hline Right cerebe & 70 & $12-74-18$ & & 0.038 & 0.054 & \\
\hline Left inferior frontal gyrus (orbitalis) & 69 & $-5226-4$ & 47 & 0.039 & 0.064 & \\
\hline \multicolumn{7}{|l|}{ (4) Left middle temporal gyrus } \\
\hline Left anterior middle temporal gyrus & 112 & $-60-6-10$ & $21 / 22$ & $0.006^{\mathrm{b}}$ & 0.001 & 6.7 \\
\hline \multicolumn{7}{|l|}{ (5) Left posterior middle temporal gyrus } \\
\hline Left inferior parietal cortex & 80 & $-32-4430$ & 40 & 0.023 & 0.000 & \\
\hline Right middle temporal gyrus & 380 & $40-680$ & 37 & $0.000^{\mathrm{b}}$ & 0.000 & \\
\hline Right middle occipital gyrus & & $30-848$ & 18 & & 0.211 & \\
\hline Left fusiform gyrus & 188 & $-40-54-8$ & 37 & $0.001^{\mathrm{b}}$ & 0.009 & \\
\hline Left hippocampus & 62 & $-30-384$ & & 0.043 & 0.025 & \\
\hline Left middle occipital gyrus & 131 & $-22-862$ & 18 & $0.004^{\mathrm{b}}$ & 0.037 & \\
\hline Left inferior occipital gyrus & & $-40-76-2$ & 19 & & 0.219 & \\
\hline Right hippocampus & 69 & $26-348$ & & 0.033 & 0.082 & \\
\hline Left middle occipital gyrus & 71 & $-30-7424$ & 19 & 0.031 & 0.164 & \\
\hline (6) Left inferior temporal gyrus & n.s. & & & & & \\
\hline
\end{tabular}

Effects are reported at a peak-level threshold of $p<0.0001$ uncorrected, $p_{\text {FWE }}<0.05$ at the cluster level, and all peaks within a cluster $>20 \mathrm{~mm}$ apart.

${ }^{a}$ Indicates that this peak was taken as a seed for the connectivity analysis.

${ }^{\mathrm{b}} \mathrm{PPI}$ results that survive a more stringent cluster-level-corrected threshold of $p_{\mathrm{FWE}}<0.008$ (to control for the six different seeds investigated).

BA, Brodman area; FWE, family-wise error; n.s., not significant; PPI, psychophysiological interaction. 
Table 2. Activation and Connectivity Effects for the Main Effect of Group

\begin{tabular}{|c|c|c|c|c|c|c|}
\hline Region & $\begin{array}{c}\text { Cluster } \\
\text { size }\end{array}$ & $x / y / z$ & $B A$ area & $\begin{array}{l}p_{F W E} \\
\text { (cluster) }\end{array}$ & $\begin{array}{l}p_{F W E} \\
(\text { peak })\end{array}$ & $T_{45}$ \\
\hline Activations & n.s. & & & & & \\
\hline \multicolumn{7}{|c|}{ (1) PPI from seed in left inferior frontal gyrus } \\
\hline Left intraparietal sulcus & 226 & $-32-4442$ & 40 & $<0.001^{\mathrm{a}}$ & 0.003 & 6.3 \\
\hline Left precentral gyrus & 93 & $-22-448$ & 6 & 0.016 & 0.022 & 5.6 \\
\hline Left supramarginal gyrus & 93 & $-56-2442$ & $40 / 3$ & 0.016 & 0.065 & 5.22 \\
\hline $\begin{array}{l}\text { Left middle occipital gyrus } \\
\text { extending into the left } \\
\text { intraparietal sulcus }\end{array}$ & 198 & $-30-8030$ & 19 & $0.001^{\mathrm{a}}$ & 0.105 & 5.03 \\
\hline Left thalamus & 63 & $-6-212$ & & 0.045 & 0.162 & 4.87 \\
\hline $\begin{array}{l}\text { (2-4) PPI from seeds in left } \\
\text { temporal pole, left anterior, } \\
\text { and middle temporal lobe }\end{array}$ & n.s. & & & & & \\
\hline \multicolumn{7}{|c|}{ (5) PPI from seed in left posterior superior/middle temporal gyrus } \\
\hline Left intraparietal sulcus & 64 & $-30-5436$ & 40 & 0.044 & 0.336 & 4.55 \\
\hline $\begin{array}{l}\text { (6) PPI from seed in left } \\
\text { inferior temporal gyrus }\end{array}$ & n.s. & & & & & \\
\hline
\end{tabular}

In all cases, the second language user group $>$ native speaker group, no significant effects for the reverse contrast. Effects are reported at a peak-level threshold of $p<0.0001$ uncorrected, $p_{\mathrm{FWE}}<0.05$ at the cluster level, and all peaks within a cluster $>20 \mathrm{~mm}$ apart.

${ }^{\text {a}} \mathrm{PPI}$ results that survive a more stringent cluster-level-corrected threshold of $p_{\mathrm{FWE}}<0.008$ (to control for the six different seeds investigated).

design. We then looked at the following contrasts: (a) the common patterns of activation for second language users and native speakers for complex sentences versus sentence format consonant strings as revealed by a conjunction between the group contrasts; (b) the differences between the two groups in processing complex sentences versus sentence format consonant strings; (c) the interaction between type of sentence structure and group. The conjunction analysis was performed using SPMs of the minimum T-statistic over two orthogonal contrasts. Inference was based on $p$-values adjusted for the search volume using the random field theory (Friston et al., 2005).

\section{Second-level model connectivity}

For the second-level connectivity analysis, we built similar models. For each seed region, we built both a simple between-groups $t$-test and a flexible factorial model. These modeled the same conditions as for the activation analysis, but on the psychophysiological interaction regressors.

For the activation as well as the connectivity analyses, we report whole-brain effects at a conservative voxellevel threshold of $p<0.0001$, family-wise error (FWE) corrected at the cluster level for multiple comparisons $(p<0.05) .{ }^{1}$ As we conduct connectivity analyses for six different seed regions, we also report which cluster-level $p$-values survive a stricter cluster-level threshold of $p_{\mathrm{FWE}}$ $<0.008$ (Tables 1 and 2). All reported coordinates are in Montreal Neurological Institute space.

\footnotetext{
${ }^{1}$ We used this conservative threshold as the current analysis is a reanalysis of a dataset. However, we want to note that no additional group differences in the activation or connectivity analyses appear at a less conservative voxel-level threshold of $p<0.001$, FWE $(p<0.05)$ corrected at the cluster level.
}

\section{Results}

\section{Performance behavioral task}

Participants were attending to the sentences as evidenced by the performance on the behavioral task. The German native speaker group spotted $85 \%$ (standard deviation $[\mathrm{SD}]=20 \%$ ) of target items in larger font size and the Dutch native speaker group $83 \%(\mathrm{SD}=19 \%)$.

\section{Activation}

(a) Conjunction (common pattern of activation across groups): The two-participant groups showed common patterns of activation for complex sentences versus consonant strings across widespread areas of a typical language network (Fig. 2 and Table 1), including left and right middle temporal cortices (on the left from very posterior parts of the temporal cortex close to occipital areas all the way to the anterior temporal pole; on the right in more anterior regions only), left inferior frontal gyrus, as well as left pre/postcentral gyrus, and left and right hippocampus.

(b) Main effect of group: There were no differences between the two groups in their activation patterns for complex sentences versus consonant strings.

(c) The interaction between type of sentence structure and group: There were no differences in the processing of the two types of sentence structures between the two groups.

\section{Connectivity}

Seed in the left inferior frontal gyrus

(a) Conjunction (common pattern of activation across groups): The task-related functional connectivity analysis from the seed in left inferior frontal gyrus showed enhanced connectivity to left occipital cortex 
Shared patterns of activation across groups

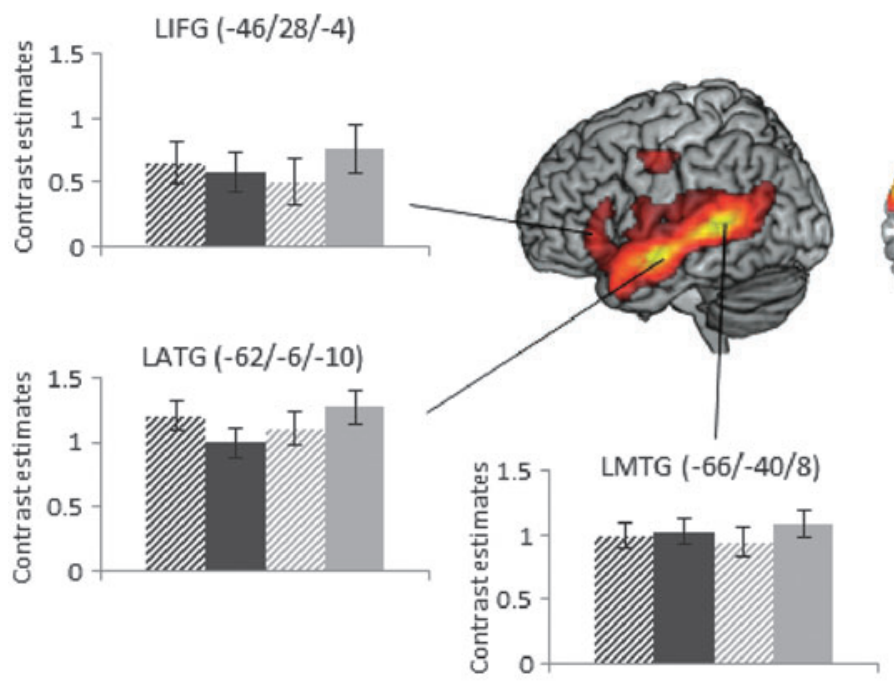

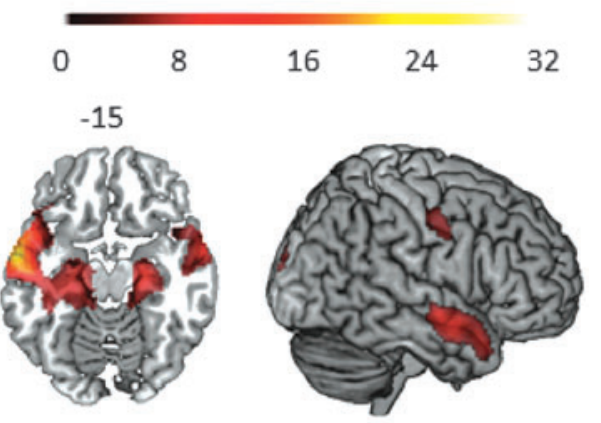

\% L2 users, Crossed Dependencies

- L2 users, Right Branching

". L1 speakers, Crossed Dependencies

n $\mathrm{L} 1$ speakers, Right Branching

FIG. 2. Conjunction of patterns of activation across the two groups for the contrast of sentences versus consonant strings, voxel-level threshold $p<0.0001$ uncorrected, cluster-level threshold $p_{\mathrm{FWE}}<0.05$ (Table 1 shows which of these psychophysiological interaction [PPI] effects survive a more stringent $p_{\mathrm{FWE}}<0.008$ cluster-level threshold to correct for number of seeds investigated). Bar graphs of contrast values with $95 \%$ confidence intervals of representative peaks are provided for illustration purposes. FWE, family-wise error. Color images available online at www.liebertpub.com/brain

and left anterior temporal cortex and right inferior frontal cortex for complex sentences compared with consonant strings for both groups, but these effects did not survive the stricter cluster-level threshold of $p_{\text {FWE }}<0.008$ (Fig. 3 and Table 1).

(b) Main effect of group: The second language user group additionally showed enhanced connectivity to left inferior parietal regions and the left middle occipital gyrus (Fig. 4 and Table 2), additional group differences in the left precentral gyrus, left supramarginal gyrus, and the left thalamus did not survive the stricter cluster-level threshold of $p_{\mathrm{FWE}}<0.008$.

(c) The interaction between type of sentence structure and group: There were no interaction effects.

\section{Seed in the left temporal pole}

(a) Conjunction (common pattern of activation across groups): The task-related functional connectivity analysis from the seed in the left temporal pole showed enhanced connectivity to the lingual gyrus and the cerebellum for complex sentences compared with consonant strings for both groups. These effects did not survive the stricter cluster-level threshold of $p_{\text {FWE }}<0.008$ (Table 1).

(b) Main effect of group: There were no differences between the groups.

(c) The interaction between type of sentence structure and group: There were no interaction effects.

\section{Seed in left anterior middle/superior temporal gyrus}

(a) Conjunction (common pattern of activation across groups): The task-related functional connectivity anal- ysis from the seed in the left anterior temporal lobe showed enhanced connectivity to regions of the language network from inferior frontal gyrus to posterior temporal cortex as well as occipital cortex gyrus for complex sentences compared with consonant strings for both groups. Most of these connectivity effects survived the stricter threshold (Table 1 and Fig. 3).

(b) Main effect of group: There were no differences between the groups.

(c) The interaction between type of sentence structure and group: There were no interaction effects.

Seed in left middle/superior temporal gyrus

(a) Conjunction (common pattern of activation across groups): The seed in left middle temporal gyrus was coupled to a slightly more anterior left middle temporal region for sentences compared with sentence format consonant strings for both groups (Table 1 and Fig. 3).

(b) Main effect of group: There were no differences between the groups.

(c) The interaction between type of sentence structure and group: There were no interaction effects.

Seed in left posterior middle/superior temporal gyrus

(a) Conjunction (common pattern of activation across groups): Enhanced connectivity to sentences compared with consonant strings from a seed in left posterior temporal gyrus was found in the left angular gyrus, right inferior temporal gyrus, and the left fusiform for both groups. Additional effects in the occipital gyrus and bilateral hippocampi did not survive the stricter cluster-level threshold of $p_{\mathrm{FWE}}<0.008$. 

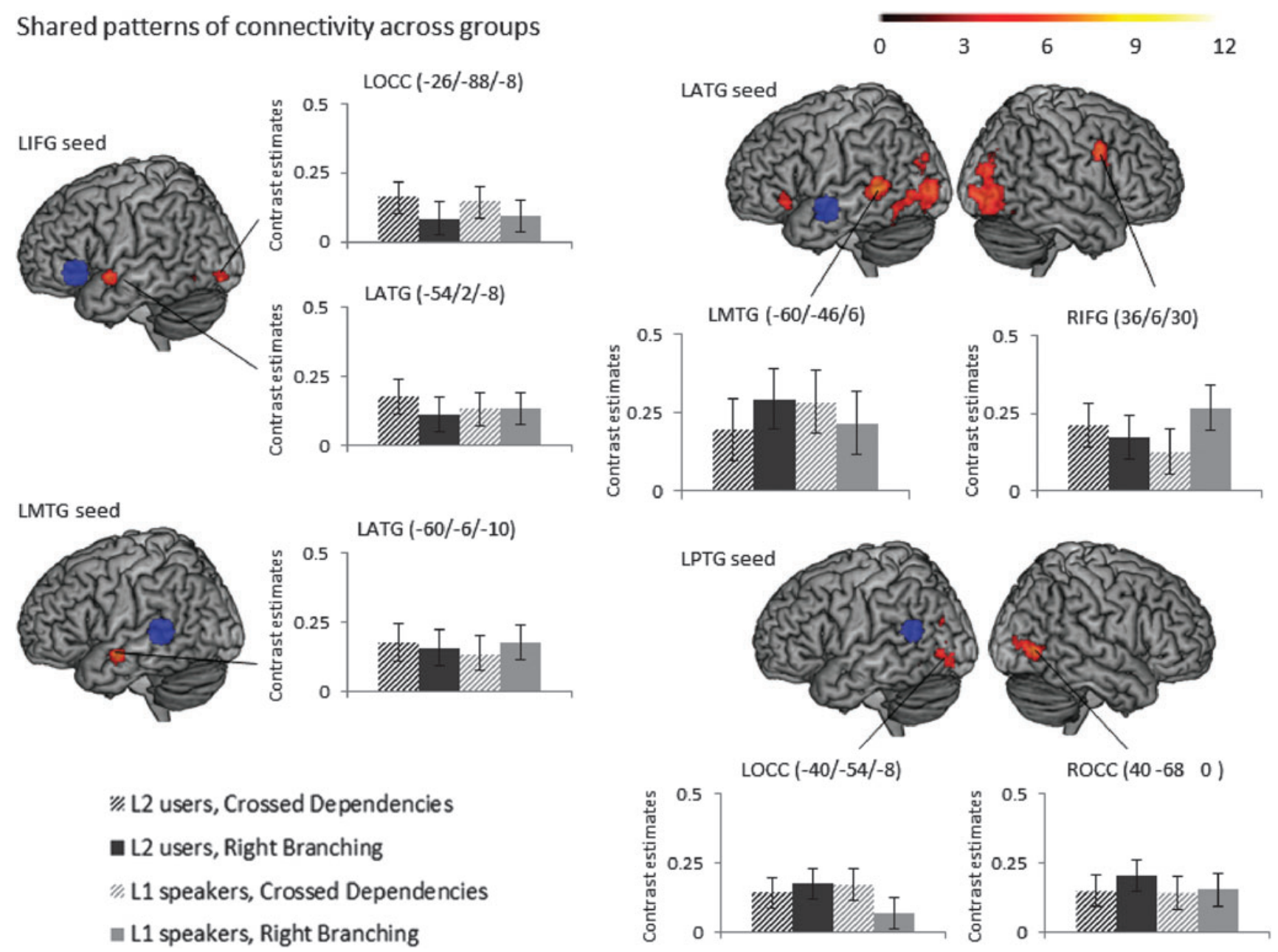

FIG. 3. Shared patterns of task-related connectivity across the two groups: Conjunction of psychophysiological interactions from seeds in LIFG, LATG, LMTG, and LPTG (seeds shown in blue), defined based on peaks in the activation analysis, see Figure 2 across the two groups for the contrast of sentences versus consonant strings, voxel-level threshold $p<0.0001$ uncorrected, and cluster-level threshold $p_{\text {FWE }}<0.05$ (Table 1 shows which of these PPI effects survive a more stringent $p_{\text {FWE }}<0.008$ cluster-level threshold to correct for number of seeds investigated). Bar graphs of contrast values with 95\% confidence intervals of representative peaks are provided for illustration purposes. LATG, left anterior temporal gyrus; LMTG, left middle temporal gyrus; LIFG, left inferior frontal gyrus; LPTG, left posterior temporal gyrus. Color images available online at www.liebertpub.com/brain

\section{Group differences in connectivity}
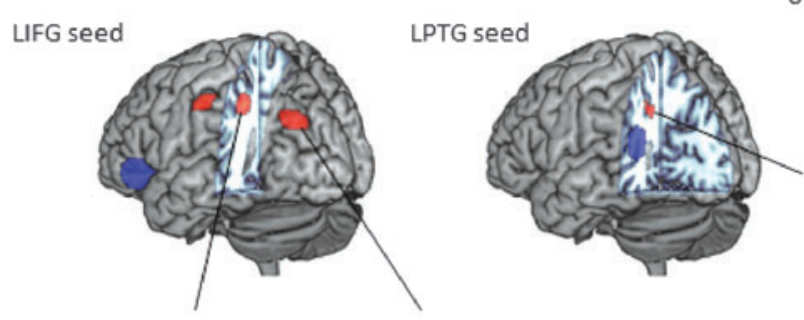

0

$\begin{array}{llll}3 & 6 & 9 & 12\end{array}$
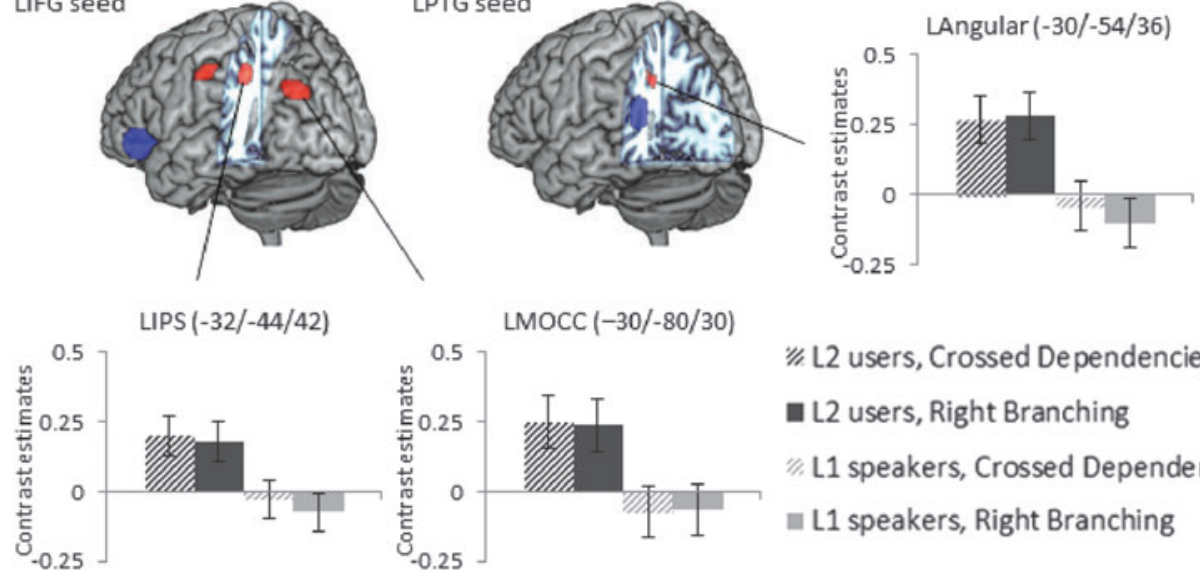

\% L2 users, Crossed Dependencies

- L2 users, Right Branching

" L1 speakers, Crossed Dependencies

- L1 speakers, Right Branching

FIG. 4. Main effect of group (German native speaker group $>$ Dutch native speaker group) of the psychophysiological interactions from seeds in LIFG and LPTG (shown in blue) for the contrast of sentences versus consonant strings, voxel-level threshold $p<0.0001$ uncorrected, and cluster-level threshold $p_{\mathrm{FWE}}<0.05$ (Table 1 shows which of these PPI effects survive a more stringent $p_{\mathrm{FWE}}<$ 0.008 cluster-level threshold to correct for number of seeds investigated). Bar graphs of contrast values with $95 \%$ confidence intervals of representative peaks are provided for illustration purposes. Color images available online at www.liebertpub.com/brain 
(b) Main effect of group: The second language user group additionally showed enhanced connectivity to a left inferior parietal region, which did not survive the stricter threshold of $p_{\mathrm{FWE}}<0.008$ (Fig. 4 and Table 2).

(c) The interaction between type of sentence structure and group: There were no interaction effects.

Seed in left inferior temporal gyrus. There were no significant connectivity effects for this seed.

\section{Discussion}

\section{Summary of the results}

In this study, we investigated the processing of complex Dutch sentences in an activation and connectivity fMRI study. We compared the brain networks of a group of Dutch native speakers and a group of German native speakers with Dutch as their second language during sentence processing. The experiments revealed the following:

(1) Activation and connectivity patterns to process complex sentences overlapped between native speakers and second language users, as revealed by conjunction analyses.

(2) The second language user group showed some additional connectivity patterns, involving areas outside the traditional language network, that is, inferior parietal regions.

\section{Connectivity and activation in the language network}

The current study revealed a large network of regions similar to those shown before for native (den Ouden et al., 2012; Griffiths et al., 2013; Snijders et al., 2009, 2010) and second language processing (Weber and Indefrey, 2009) for similar manipulations for simpler sentences. As expected, the core of these networks resides in the left middle temporal and inferior frontal cortex. In the context of sentence-level processing, these are thought to be linked to key language processing functions, namely the access to the relevant building blocks such as words, from memory and the unification of these into a coherent message-level representation both on the semantic and syntactic levels, respectively (Hagoort, 2005, 2014; Tyler et al., 2011). Not surprisingly, these areas also show enhanced connectivity during sentence processing as they have to work in consort during online processing.

Anatomically, these frontal and temporal areas of the language network are connected by several fiber bundles, such as the arcuate fasciculus, connecting the inferior frontal cortex with parts of the posterior temporal cortex, and the uncinate, connecting frontal cortex and anterior temporal cortex (Friederici and Gierhan, 2013; Hagoort, 2014). In addition to the key network on the left, we found additional activations in the right temporal gyrus. While not typically involved, for example, in syntactic processing (Segaert et al., 2012), one should keep in mind that in this study we are investigating a broad contrast of (complex) sentences versus sentence format consonant strings, which thus incorporates all kinds of levels of language processing (sentence and word-level syntax and semantics, lexical processing, and control processes), which do engage these areas (Hagoort, 2014). The involvement of a large network of areas, includ- ing those outside of left inferior frontal and temporal gyrus, is thus not surprising.

Both for the activation and the connectivity analyses, the vast overlap between these language networks for second language users and native speakers is evident. Thus, even for highly complex and even previously unknown sentence structures, the main processing network for a second language seems to be shared with that of a native language at least at the high-proficiency level of the present L2 user group. This also supports the electrophysiological findings by Davidson and Indefrey (2009), which showed that these structures can be learned quickly. This also mirrors previous findings in the literature for simpler sentence structures (Indefrey, 2006; Luke et al., 2002; Perani et al., 1998; Weber and Indefrey, 2009) and provides evidence against ideas that a second language is processed by a fundamentally different brain network.

\section{Less automatic processing in second language users}

Several areas showed increased connectivity patterns in the second language user group for processing complex sentence structures compared with the native speakers. This suggests that some additional or different mechanisms are at play to process complex sentences in a non-native language. The increase in connectivity in this group from left inferior frontal gyrus and less strongly from left posterior temporal gyrus to inferior parietal regions could potentially stem from increased cognitive demands on the multidemand and control system (Duncan, 2010; Humphreys and Lambon Ralph, 2015) when processing a second language. The observation that processing a second language, even at a high-proficiency level, could lead to increased demands on cognitive control mechanisms has been claimed before (Abutalebi et al., 2008; Green and Abutalebi, 2013). This suggests that there are no differences in the organization of the core language machinery itself, which appears to be shared between a first and a second language just as previous research suggests (Weber and Indefrey, 2009).

The differences arise in the increased demands on systems that act on the core language processing system to ensure that the task demands (efficiently parsing the sentence and getting to a coherent message level representation) are met. These cognitive demands increase when processing a second language and the result is an increase in connectivity in the frontoparietal network related to top-down cognitive control and attention, as well as executive semantic decisions (Humphreys and Lambon Ralph, 2015). Specifically, the connectivity differences seem to be involving the left intraparietal sulcus part close to the angular gyrus, which a recent meta-analysis in which high- versus low-demand semantic tasks were contrasted had also found (Noonan et al., 2013) and linked to domain-general executive control demands.

Generally, our findings seem to indicate that the core language machinery is shared between native and second language user groups, even when processing highly complex structures that do not exist in the L2 users' native language. With an increase in cognitive control, these areas can handle second language structures as effectively as an L1 group. The differences in brain networks are thus only in peripheral areas related to cognitive control, which are influencing the core network. We thus find no evidence for a fundamental 
distinction between first and second language processing, as in more procedural versus declarative processing as claimed by Ullman (2001a,b) or a specific difference in grammatical processing of nonlocal dependencies in a second language (Clahsen and Felser, 2006).

\section{No specific group differences for later learned structures}

Next to investigating general differences between language processing in native speakers and second language users, we wanted to see whether there are any specific processing differences related to later learned structures that cannot be easily mapped on an existing structure from the native language. At the current conservative statistical thresholds, we do not see any such differences indicating that later learned sentence structure can also be integrated into existing language networks in the brain. There is thus no concrete evidence with this analysis for different language mechanisms in processing these later learned sentence structures. However, subtle difference in organization within these overlapping language networks that were indicated by the repetition effects analysis of the same dataset (see Weber's (2012) $\mathrm{PhD}$ thesis) should be further investigated in future studies. Furthermore, future investigations should see whether the current results hold under different task conditions, for example, when sentence comprehension questions are asked, as well as for other, less closely related language combinations.

\section{Conclusion}

In sum, while cortical language networks, involving left inferior frontal and left middle temporal regions, largely overlapped between second language users and native speakers, nontraditional language areas in inferior parietal regions show connectivity differences, hinting at less automatic processing of an L2, thus requiring more cognitive control.

\section{Acknowledgments}

This research was supported by an NWO Toptalent (Grant No. 021.001.007) to K.W. The authors would like to thank Monique Flecken for helpful feedback on the manuscript.

\section{Author Disclosure Statement}

No competing financial interests exist.

\section{References}

Abutalebi J, Annoni JM, Zimine I, Pegna AJ, Seghier ML, LeeJahnke H, Lazeyras F, Cappa SF, Khateb A. 2008. Language control and lexical competition in bilinguals: an event-related FMRI study. Cereb Cortex 18:1496-1505.

Bach E, Brown C, Marslen-wilson W. 1986. Crossed and nested dependencies in German and Dutch: a psycholinguistic study. Lang Cogn Process 1:249-262.

Clahsen H, Felser C. 2006. How native-like is non-native language processing? Trends Cogn Sci 10:564-570.

Consonni M, Cafiero R, Marin D, Tettamanti M, Iadanza A, Fabbro F, Perani D. 2013. Neural convergence for language comprehension and grammatical class production in highly proficient bilinguals is independent of age of acquisition. Cortex 49:1252-1258.
Dallas A, Kaan E. 2008. Second language processing of fillergap dependencies by late learners. Lang Linguist Compass 2:372-388.

Davidson DJ, Indefrey P. 2009. Plasticity of grammatical recursion in German learners of Dutch. Lang Cogn Process 24:1335-1369.

den Ouden D-B, Saur D, Mader W, Schelter B, Lukic S, Wali E, Timmer J, Thompson CK. 2012. Network modulation during complex syntactic processing. Neuroimage 59:815-823.

Duncan J. 2010. The multiple-demand (MD) system of the primate brain: mental programs for intelligent behaviour. Trends Cogn Sci 14:172-179.

Friederici AD, Gierhan SME. 2013. The language network. Curr Opin Neurobiol 23:250-254.

Friston KJ, Penny WD, Glaser DE. 2005. Conjunction revisited. Neuroimage 25:661-667.

Green DW, Abutalebi J. 2013. Language control in bilinguals: the adaptive control hypothesis. J Cogn Psychol (Hove) 25:515-530.

Griffiths JD, Marslen-Wilson WD, Stamatakis EA, Tyler LK. 2013. Functional organization of the neural language system: dorsal and ventral pathways are critical for syntax. Cereb Cortex 23:139-147.

Grodzinsky Y, Friederici AD. 2006. Neuroimaging of syntax and syntactic processing. Curr Opin Neurobiol 16:240-246.

Hagoort P. 2005. On Broca, brain, and binding: a new framework. Trends Cogn Sci 9:416-423.

Hagoort P. 2014. Nodes and networks in the neural architecture for language: Broca's region and beyond. Curr Opin Neurobiol 28:136-141.

Hagoort P, Indefrey P. 2014. The neurobiology of language beyond single words. Annu Rev Neurosci 37:347-362.

Hartsuiker RJ, Pickering MJ, Veltkamp E. 2004. Is syntax separate or shared between languages?: cross-linguistic syntactic priming in Spanish-English bilinguals. Psychol Sci 15:409-414.

Humphreys GF, Lambon Ralph MA. 2015. Fusion and fission of cognitive functions in the human parietal cortex. Cereb Cortex 25:3547-3560.

Indefrey P. 2006. A meta-analysis of hemodynamic studies on first and second language processing: which suggested differences can we trust and what do they mean? Lang Learn 56:279-304.

Kaan E, Vasić N. 2004. Cross-serial dependencies in Dutch: testing the influence of NP type on processing load. Mem Cognit 32:175-184.

Luke K-K, Liu H-L, Wai Y-Y, Wan Y-L, Tan LH. 2002. Functional anatomy of syntactic and semantic processing in language comprehension. Hum Brain Mapp 16:133-145.

McLaren DG, Ries ML, Xu G, Johnson SC. 2012. A generalized form of context-dependent psychophysiological interactions (gPPI): a comparison to standard approaches. Neuroimage 61:1277-1286.

Menenti L, Gierhan SM, Segaert K, Hagoort P. 2011. Shared language: overlap and segregation of the neuronal infrastructure for speaking and listening revealed by functional MRI. Psychol Sci 22:1173-1182.

Nieuwland MS, Van Berkum JJA. 2006. Individual differences and contextual bias in pronoun resolution: Evidence from ERPs. Brain Res 1118:155-167.

Noonan KA, Jefferies E, Visser M, Lambon Ralph MA. 2013. Going beyond inferior prefrontal involvement in semantic control: evidence for the additional contribution of dorsal angular gyrus and posterior middle temporal cortex. J Cogn Neurosci 25:1824-1850. 
Nowak MA, Komarova NL, Niyogi P. 2002. Computational and evolutionary aspects of language. Nature 417:611-617.

Perani D, Paulesu E, Galles NS, Dupoux E, Dehaene S, Bettinardi V, Cappa SF, Fazio F, Mehler J. 1998. The bilingual brain. Proficiency and age of acquisition of the second language. Brain 121:1841-1852.

Schoonbaert S, Hartsuiker RJ, Pickering MJ. 2007. The representation of lexical and syntactic information in bilinguals: evidence from syntactic priming. J Mem Lang 56:153-171.

Sebastian R, Laird A, Kiran S. 2011. Meta-analysis of the neural representation of first language and second language. Appl Psycholinguist 32:799-819.

Segaert K, Menenti L, Weber K, Petersson KM, Hagoort P. 2012. Shared syntax in language production and language comprehension-an FMRI study. Cereb Cortex 22:16621670 .

Snijders TM, Petersson KM, Hagoort P. 2010. Effective connectivity of cortical and subcortical regions during unification of sentence structure. Neuroimage 52:1633-1644.

Snijders TM, Vosse T, Kempen G, Van Berkum JJA, Petersson KM, Hagoort P. 2009. Retrieval and unification of syntactic structure in sentence comprehension: an fMRI study using word-category ambiguity. Cereb Cortex 19:1493-1503.

Stromswold K, Caplan D, Alpert N, Rauch S. 1996. Localization of syntactic comprehension by positron emission tomography. Brain Lang 52:452-473.

Tyler LK, Marslen-Wilson WD, Randall B, Wright P, Devereux BJ, Zhuang J, Papoutsi M, Stamatakis EA. 2011. Left inferior frontal cortex and syntax: function, structure and behaviour in patients with left hemisphere damage. Brain 134:415-431.
Uddén J, Ingvar M, Hagoort P, Petersson KM. 2012. Implicit acquisition of grammars with crossed and nested non-adjacent dependencies: investigating the push-down stack model. Cogn Sci 36:1078-1101.

Ullman MT. 2001a. The neural basis of lexicon and grammar in first and second language: the declarative/procedural model. Bilingualism Lang Cogn 4:105-122.

Ullman MT. 2001b. A neurocognitive perspective on language: the declarative/procedural model. Nat Rev Neurosci 2:717-726.

Ullman MT. 2006. Is Broca's area part of a basal ganglia thalamocortical circuit? Cortex 42:480-485.

Weber K. 2012. The language learning brain: evidence from second language learning and bilingual studies of syntactic processing. PhD, Radboud University, Nijmegen.

Weber K, Indefrey P. 2009. Syntactic priming in German-English bilinguals during sentence comprehension. Neuroimage 46:1164-1172.

Zwart J-W. 1996. Verb clusters in Continental West Germanic dialects. In: Black JR, Motapanyane V (eds.) Microparametric Syntax and Dialect Variation. Amsterdam/Philadelphia, PA: John Benjamins Publishing Company; pp. 229-258.

Address correspondence to: Kirsten Weber Max Planck Institute for Psycholinguistics P.O. Box 310 6500 AH Nijmegen The Netherlands

E-mail: kirsten.weber@mpi.nl 